\title{
Ultrasound-guided injection of platelet-rich plasma (PRP) in rotator cuff tendinopathy: effect on patients' symptoms and supraspinatus tendon thickness
}

\author{
Gamal Eldine Niazi ${ }^{1 *}$ D, Mohammed Sobhi Hassan ${ }^{1}$ and Dalia M. Elfawy ${ }^{2}$
}

\begin{abstract}
Background: Rotator cuff tendinopathy is considered a major cause of shoulder pain and disability that is increasing substantially with age affecting more than half of the general population by the age of 60 years. This study aims to assess the effect of ultrasound-guided injection of platelet-rich plasma on patient symptoms and supraspinatus tendon thickness in cases of rotator cuff tendinopathy.

Results: This was a single-arm interventional study conducted on 30 patients with age ranging between 27 and 54 years old. Following US-guided injection of PRP, patients were evaluated clinically using the Shoulder Pain and Disability Index (SPADI) scoring system and radiologically using ultrasonographic supraspinatus tendon thickness measurements at 4, 8, 12, and 24 weeks. Our study showed remarkably noticeable changes when comparing the preinjection and post-injection SPADI scoring system. There is highly statistically significant pain and disability score and percentage improvement, yet on the other hand, the radiological improvement shows no statistically significant difference found between baseline tendon thickness and its follow-up at 4, 8, and 12 weeks while only there was a statistically significant decrease in tendon thickness found at 24 weeks with $P$ value $=0.043$.

Conclusions: The ultrasound-guided PRP injection for supraspinatus tendinopathy cases is a safe, cheap, and easily prepared outpatient procedure which showed competitive, promising, and well-proved results when compared to other modality outcomes such as conventional surgeries, arthroscopic procedures, and physiotherapy.
\end{abstract}

Keywords: Ultrasound-guided injection, Platelet-rich plasma, Rotator cuff tendinopathy

\section{Background}

Rotator cuff tendinopathy has been considered a major cause of shoulder pain and disability that is increasing substantially with age affecting more than half of the general population by the age of 60 years [1].

Being responsible for $85 \%$ of the cases, rotator cuff tendinopathy is considered the most common cause of shoulder pain and disability [2].

The supraspinatus muscle is the most commonly injured in rotator cuff tendinopathy, and most of the time,

\footnotetext{
* Correspondence: gamalniazi@yahoo.com

${ }^{1}$ Radiology Department, Ain Shams University, Cairo, Egypt

Full list of author information is available at the end of the article
}

it is accompanied with another rotator cuff muscle tendinopathy [3].

The main risk factors for such cases include overweight, old age, repetitive lifting, or overhead activities [4].

Shoulder ultrasound plays a major role in the diagnosis of rotator cuff tendinopathy having the advantages of being readily available, highly sensitive, relatively inexpensive, and non-invasive. However, it has the disadvantages of being operator dependent and requiring standardized scanning technique [5].

The management of rotator cuff tendinopathy nowadays includes oral drugs (like NSAIDs), local injections with corticosteroids, and physical therapy or lastly 
surgical repair depending on the type and extent of the injury, yet it is associated with a high risk of infection and damage to surrounding nerves and blood vessels with up to 6 months of recovery period [6].

Recently, there are numerous well-documented studies about using blood and its products to facilitate the healing course and augmenting musculoskeletal repair [7].

Platelet-rich plasma (PRP) is a whole blood product containing great concentrations of platelets that release different kinds of growth mediators with restorative properties. These platelet-derived growth factors include transforming growth factor (TGF-B), which is concentrated in collagen synthesis, and vascular endothelial growth factor (VEGF), which aids to induce endothelial cell multiplying and migration and stimulates cell mitosis [8].

Despite the increased use of PRP in the management of different MSK disorders, only few studies have searched the value of PRP injection in cases of rotator cuff tendinopathy [9].

This study aims to assess the effect of ultrasoundguided injection of platelet-rich plasma (PRP) on patient symptoms and supraspinatus tendon thickness in cases of rotator cuff tendinopathy.

\section{Methods}

This was a single-arm interventional study conducted in the period between October 2018 and September 2019; 30 patients were included in the study ranging from 27 to 54 years old (Fig. 1).

\section{Inclusion criteria}

Patients complaining of shoulder pain with the following criteria:

1. Diagnosed with rotator cuff tendinopathy (supraspinatus tendinosis) by musculoskeletal ultrasound

2. Symptoms have been continuous for more than 3 months and failed conservative treatment of at least 4 weeks of formal medical and physical therapy

\section{Exclusion criteria}

1. Prior surgery to the injured shoulder

2. Full-thickness rotator cuff tear

3. Current treatment with anticoagulation

4. Steroid injection in the past 6 months in the injured shoulder

Patient enrollment in the study $(n=30)$ - Full history taking- Full clinical examination- Shoulder ultrasound examination- Clinical evaluation using SPADI scoring system

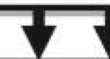

Written informed consent

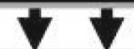

4 weeks of conservative management

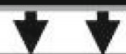

Preparation of Platelet Rich Plasma (PRP)

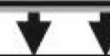

Ultrasound guided injection of PRP

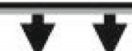

Clinical evaluation using SPADI scoring system and supraspinatous tendon thickness measurement by ultrasound at 4,8,12 and 24 weeks post PRP injection

Fig. 1 Flowchart for study methodology 
5. Prior PRP treatment to the injured shoulder

6. Bleeding disorders or preoperative platelet count less than 50,000

7. Presence of another disease that may cause shoulder pain and dysfunction as rheumatoid arthritis

\section{Ethical considerations}

The whole procedures are explained in detail to the patients, and they received detailed written information which explains the entire procedure and any possible risks that may happen (informed consent).

\section{Study tools}

1. A centrifuge machine to obtain the platelet-rich plasma

2. An ultrasound machine with superficial probe 10 $\mathrm{Hz}$

3. Syringes $3 \mathrm{~cm}, 10 \mathrm{~cm}$, and $20 \mathrm{~cm}$

4. Ten-centimeter sterile test tubes

5. Citrate dextrose anticoagulant

6. Spinal needle

7. Antiseptic conditions

8. Lidocaine $2 \%$ (local anesthetic)

\section{Study procedures}

\section{Patient preparation}

Patients were subjected to the following:

- Full history taking including gender, age, right-sided or left-sided dominance, affected shoulder, history of occupation requiring shoulder overuse and night pain, history of bleeding disorder, history of prior surgeries or steroid injections

- Full clinical examination

- Shoulder ultrasound examination

- Explaining the whole procedure including the steps, outcomes, and complications

- Written consents were taken from the patients according to ethical committee considerations

- Patients are evaluated clinically using the Shoulder Pain and Disability Index (SPADI) scoring system and radiologically using ultrasonographic supraspinatus tendon thickness measurements in each visit

\section{Procedure}

\section{A.) Pre-procedure instructions}

Patients who have scheduled an injection should stop all anti-inflammatory medications for 1 week prior to injection. Patients should be prepared to take it easy for about 2-3 days after the injection.

\section{B.) Preparation of platelet-rich plasma (PRP)}

PRP was prepared using standard techniques. Patient blood was collected under aseptic conditions in acid citrate dextrose tubes. An antecubital blood draw of $20 \mathrm{ml}$ was done. This blood was put in a centrifuge at 2000 rotation per minute (soft spin). The whole blood is separated into three layers. The supernatant layer of plasma and buffy coat were separated and subjected to centrifugation at 3000 rotation per minute (hard spin). In the final end product, the upper two thirds of the tube will be containing platelet-poor plasma which is removed, and the lower one third will be PRP enhanced with a superficial buffy coat which will be used for injection [10].

\section{C.) Technique of the procedure}

Each participant underwent a single injection of PRP. The procedure starts with a diagnostic ultrasound to detect the site of the subacromion bursa. Then the patient is positioned in a sitting position, and the site of injection is decided (Fig. 2). Under aseptic conditions, sterile surgical gloves, probe cover, sterile gel, and betadine were used to sterilize the site of injection and probe surface. Three milliliters of local anesthesia is given at the injection site subcutaneously. Using a 22-gauge spinal needle, $5-7 \mathrm{ml}$ of the PRP product was injected at the subacromion bursa of the affected site, and under ultrasound guidance (Fig. 3).

After injection, the patient's arms were supported with a simple sling for $48 \mathrm{~h}$. Patients were requested to mobilize the shoulder at the earliest.

D.) Aftercare following PRP injection

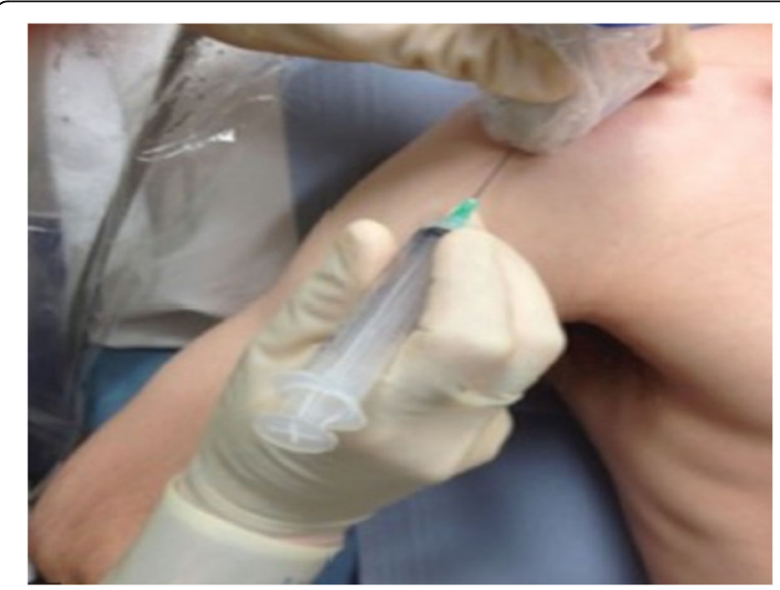

Fig. 2 Patient positioning during ultrasound-guided injection 


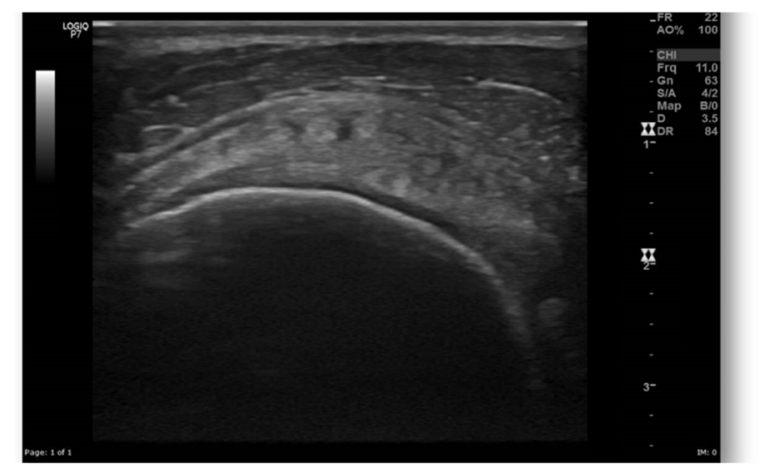

(A)

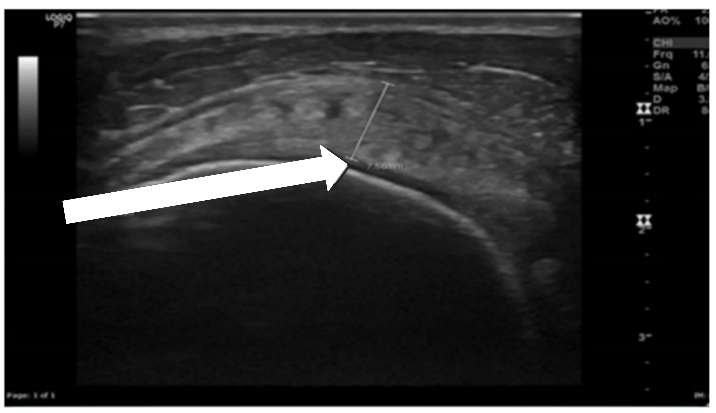

(B)

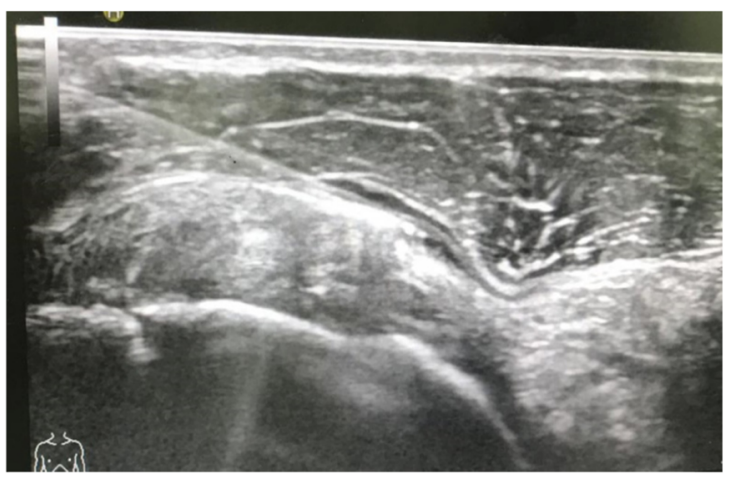

(C)

Fig. 3 a Right shoulder ultrasound with diffuse supraspinatus thickening and heterogenicity. $\mathbf{b}$ Baseline supraspinatus tendon thickness (white arrow). $\mathbf{c}$ Ultrasound-guided injection of PRP in the right subacromion bursa

- Participants were advised to use relative rest including 2 days off work.

- Patients should not take anti-inflammatory medications for the next 2 weeks.

- Slowly advance activities of daily living progressively over 2 weeks and return to activity as tolerated.

- Pain in the area of injection is common. Pain pills in the form of paracetamol pills are provided for this. It usually resolves in the first few days.
- A follow-up post-procedure exam will be scheduled after about 4, 8, 12, and 24 weeks.

- If the patient feels any of the following symptoms, immediately, he must contact our department: prolonged redness, swelling, fevers, and chills.

\section{E.) Outcome measures}

We use two types of measures which are the Shoulder Pain and Disability Index (SPADI) score and the ultrasound measure of the supraspinatus tendon. The SPADI score is a questionnaire answered by the patient to assess the severity of pain and on doing some ordinary everyday tasks and how difficult they are done on a scale of $0-10$. Then results are calculated to get scores ready to be compared with after treatment (Fig. 4) [11].

For the supraspinatus tendon thickness measurement, the arm was placed into the modified crass position that is the palm of the hand on the iliac crest with the elbow directed posteriorly. On-screen calipers were used to measure between the echogenic tendon boundaries at a point $20 \mathrm{~mm}$ medial to the insertional site with the mean of these measures used for analysis. The mean average tendon thickness in the adult is about $6.6 \mathrm{~mm}$.

\section{Results}

This study was conducted on 30 cases with age ranged from 27 to 54 years and with mean \pm SD of $39.8 \pm 9.1$ years. They were 10 females (33.3\%) and 20 males (66.7\%). The majority of the cases (20 cases) had rightsided affection, 18 of which were right-sided dominant, and the other (10 cases) cases were left-sided affected, 6 of them were left-sided dominant.

\section{Outcome measures \\ SPADI score (Table 1)}

For the whole study group members, an exploratory analysis of SPADI scores was done prior to the procedure and then at 4, 8, 12, and 24 weeks after the injection. Data of 30 patients were analyzed. The total scoring difference between pre-injection and post-injection shows that there was a statistically significant decrease in the SPADI score at the different points of follow-up $(P<0.01)$ (Fig. 5).

Based on the previous results, a statistically significant improvement of the total scores and their percentages should be concluded. They showed a remarkable drop from a mean value \pm SD of $98.87 \pm 8.70(88-116)$ (baseline values) to $72.67 \pm 4.91(67-82)$ after 24 weeks (postinjection), completing its descending way. The $P$ value of $<0.001$ suggested high statistical significance. However, the maximum mean difference between the four points 


\section{SHOULDER PAIN AND DISABILITY (SPADI)}

Name: Date:

Instructions:

Please answer the following questions by writing a number from 0-10 in the blank provided. If you feel a questions does not pertain to you please put a NA (not applicable) in the space. We will ask you to repeat this index in order to help our facility keep track of our treatment outcomes.

\begin{tabular}{|c|c|}
\hline \multicolumn{2}{|c|}{$\begin{array}{l}\text { Pain scale: On a scale of } 0-10, \text { How severe is your pain: } \\
0=\text { "no pain at all" }\end{array}$} \\
\hline 1. At its worst? & \\
\hline 2. When lying on the involved side? & \\
\hline 3. Reaching for something on a high shelf? & \\
\hline 4. Touching the back of your neck? & \\
\hline 5. Pushing with the involved arm & \\
\hline \multicolumn{2}{|c|}{$\begin{array}{l}\text { Disability scale: On a scale of 0-10, How much difficulty do you have: } \\
0=\text { "no difficulty"----10 = "so difficult it required help" }\end{array}$} \\
\hline 1. Washing your hair? & \\
\hline 2. Washing your back? & \\
\hline 3. Putting on an undershirt or pullover sweater? & \\
\hline 4. Putting on a shirt that buttons down the front? & \\
\hline 5. Putting on your pants? & \\
\hline 6. Placing on object on a high shelf? & \\
\hline 7. Carrying a heavy object of 10 pounds? & \\
\hline 8. Removing something form your back pocket? & \\
\hline
\end{tabular}

\section{To be completed bv office staff:}

Circle one: Initial / Re-eval / Discharge Diagnosis:

Total \# of treatments:

Pain Scale Score:

Disability Scale Score:

Total Score:

[Scoring: Summate the scores and divide by the highest score possible (130 if all questions answered). If an item is deemed not applicable, no score is calculated. Multiply the total score by 100.]

Roach KE, Budiman-Mak E, Songsirdej N, Lertratanakul Y. Development of a shoulder pain and disability index. Arthritis Care Res 1991:4:143-9.

Arthritis Care Res 1991:4:143-9.

Virginia: Learn Publications.

Fig. 4 Shoulder pain and disability index (SPADI)

Table 1 Comparison between the baseline SPADI score and its follow-up at 4, 8, 12, and 24 weeks

\begin{tabular}{|c|c|c|c|c|c|c|}
\hline & \multicolumn{3}{|l|}{ SPADI score } & \multicolumn{3}{|c|}{ Paired $\boldsymbol{t}$ test } \\
\hline & $\overline{M e a n} \pm$ SD & Range & Difference $^{a}$ & Test value & $\boldsymbol{P}$ value & Sig. \\
\hline aseline & $98.87 \pm 8.70$ & $88-116$ & - & - & - & - \\
\hline 4 weeks & $91.73 \pm$ & 82 & 7 & 1 & م & $\mathrm{HS}$ \\
\hline weeks & $85.00 \pm 6.35$ & $76-97$ & $6.73 \pm 1.58$ & 15.073 & 0.000 & $\mathrm{HS}$ \\
\hline eks & $76.07=$ & $70-89$ & 2.87 & 16.310 & 0.000 & HS \\
\hline t weeks & $72.67 \pm 4.91$ & $67-82$ & $3.40 \pm 1.40$ & 18.430 & 0.000 & $\mathrm{HS}$ \\
\hline
\end{tabular}

$P$ value $>0.05$ : nonsignificant; $P$ value $<0.05$ : significant; $P$ value $<0.01$ : highly significant

${ }^{\mathrm{a}}$ Difference was calculated from the previous reading of follow-up is noted throughout the duration between 8 and 12 weeks post-injection which was $85.00 \pm 6.35$ at 8 weeks and $76.07 \pm 5.70$ at 12 weeks post-injection.

\section{Tendon thickness (Table 2)}

The previous table shows that there was no statistically significant difference found between baseline tendon thickness and its follow-up at 4, 8, and 12 weeks while only there was a statistically significant decrease in tendon thickness found at 24 weeks with $P$ value $=0.043$ (Fig. 6).

The previous graph shows that the radiological changes as regards the tendon thickness appear only at the last follow-up visit after 24 weeks. So, clinical improvement precedes the radiological improvement.

There were no adverse events or unexpected side effects as regards the PRP injection except for the tolerable pain. Twenty-four cases reported that they were 


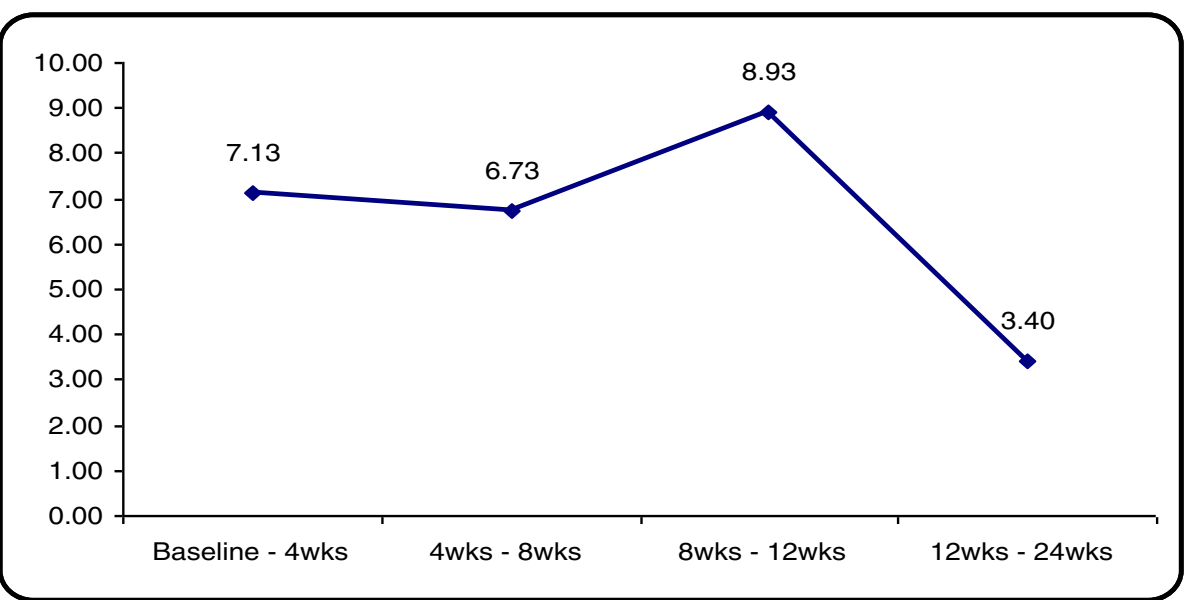

Fig. 5 A graph displaying the difference in the SPADI score between the four points of follow-up visits

completely satisfied with care, yet the other 6 cases were slightly satisfied.

Immediate post-treatment pain was recorded. The clinical impression was that pain as a result of the injection was modest and spontaneously resolved in 2 to 3 days.

\section{Discussion}

Rotator cuff injuries are responsible for approximately $85 \%$ of cases of shoulder pain and disability [2].

It has been hypothesized that improving angiogenesis and augmenting tissue remodeling by inducing the release of fundamental growth factors (GFs) such as vascular endothelial growth factor (VEGF-B) via PRP administration can positively affect healing process [7]. The theory supporting the use of PRP in treating various musculoskeletal conditions is based on the concept of reparative formation. In this context, platelets play an essential role in the healing process through the normal secretion of GFs and recruitment of reparative cells [11].

Our study showed remarkably noticeable change when comparing the pre-injection and post-injection SPADI scoring system at $4,8,12$, and 24 weeks. There is highly

Table 2 Comparison between baseline tendon thickness and its follow-up at 4 weeks, 8 weeks, 12 weeks, and 24 weeks

\begin{tabular}{lllllllll}
\hline & \multicolumn{3}{l}{ Tendon thickness in $\mathrm{mm}$} & \multicolumn{3}{l}{ Paired $\boldsymbol{t}$ test } \\
\cline { 2 - 3 } & Mean \pm SD & Range & Difference $^{\mathbf{a}}$ & & Test value & $\boldsymbol{P}$ value & Sig. \\
\hline Baseline & $7.26 \pm 0.44$ & $6.7-8.1$ & - & & & & \\
4 weeks & $7.22 \pm 0.35$ & $6.7-8.1$ & $0.04 \pm 0.10$ & 0.276 & 0.784 & NS \\
8 weeks & $7.18 \pm 0.43$ & $6.7-8.1$ & $0.02 \pm 0.05$ & 0.504 & 0.618 & NS \\
12 weeks & $7.17 \pm 0.50$ & $6.7-8.1$ & $0.01 \pm 0.10$ & 0.523 & 0.604 & NS \\
24 weeks & $7.10 \pm 0.30$ & $6.6-7.6$ & $0.07 \pm 0.37$ & 2.221 & 0.043 & S \\
\hline
\end{tabular}

$P$ value $>0.05$ : nonsignificant; $P$ value $<0.05$ : significant; $P$ value $<0.01$ : highly significant

${ }^{\mathrm{a}}$ Difference was calculated from the previous reading statistically significant pain and disability score improvement, yet on the other hand, the radiological improvement showed no statistically significant difference found between baseline tendon thickness and its follow-up at 4,8 , and 12 weeks while only there was a statistically significant decrease in tendon thickness found at 24 weeks with $P$ value $=0.043$.

There are few studies that suggested the efficacy of ultrasound-guided PRP injection on rotator cuff tendinopathy. One study closely resembled ours [12]. Also, other studies explored the use of PRP injection in augmentation to arthroscopic rotator cuff repair with intra-operative PRP injection [13].

Some studies also compared between PRP injection and physiotherapy in the treatment of chronic supraspinatus muscle partial tear [14].

Regarding the side effects of the procedure; our study-similar to the Sengodan group study-showed that the most common side effect was post-injection pain which was modest and spontaneously resolved in 2 to 3 days. There were no other specific side effects regarding PRP injections [10].

Mautner et al. stated that PRP injections almost carry no risk of acquiring a transmitted blood-borne infection or causing any anaphylactic reaction [15].

Randelli et al. stated that all his patients had reduction in pain, functional improvement, with no adverse effect when using PRP for the augmentation of arthroscopy in the treatment of cuff repairs. This was shown by the improvement in the constant score at 12 weeks following the repair [16].

Saltzman et al. concluded that there is an improvement in pain and reduction in the rehabilitation period in cases where PRP augmentation was done intraoperatively during arthroscopy for patients with rotator cuff tear. When PRP is used to augment rotator cuff 


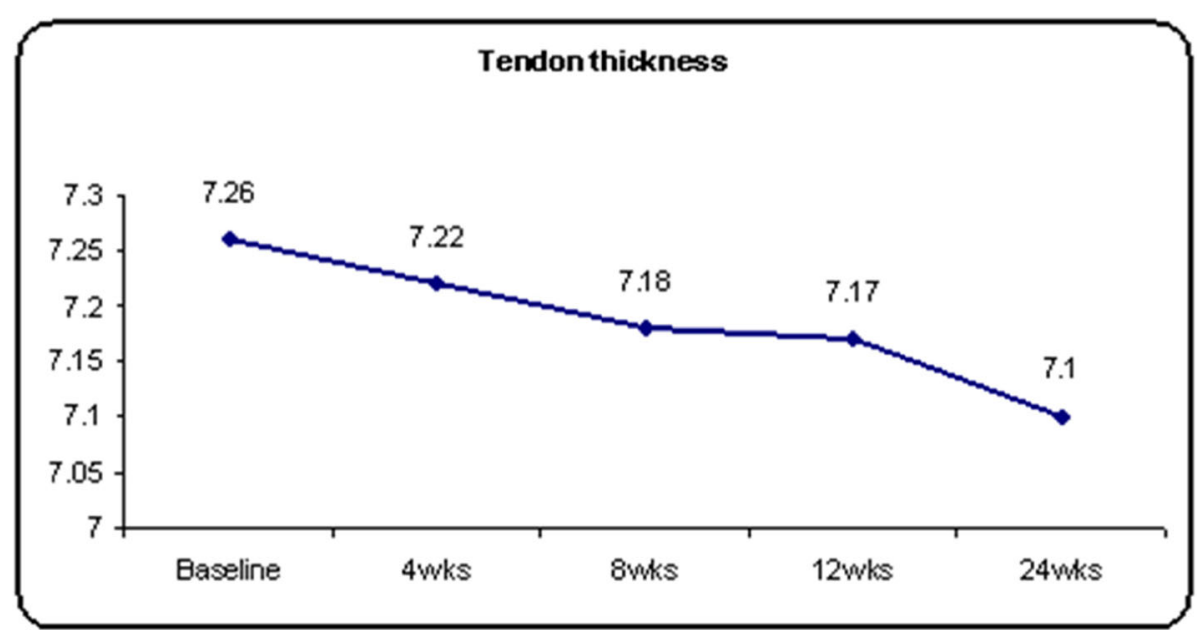

Fig. 6 A graph displaying the difference in the tendon thickness throughout the points of follow-up

repair, it resulted in decreased re-tear rates, early going back to day-to-day activity, and improvement in pain [17].

IIhanli et al. compared between PRP therapy and physiotherapy in the treatment of supraspinatus tendinopathy and conclude that PRP therapy is a well-tolerated therapeutic application that showed encouraging clinical results in patients with chronic tendinopathy and may be as effective as physiotherapy [14].

Parada et al. suggested that surgery, as a classic option for treating these cases, has risks such as infection and damage to surrounding nerves and blood vessels with up to 6 months of recovery period depending on the severity of the injury. Stiffness, weakness, chronic pain, or incomplete healing after surgery can occur [18].

In a double-blinded randomized control trial, Schwitzguebel et al. stated that PRP injections within interstitial supraspinatus tears did not improve tendon healing or clinical scores compared with saline injections and were associated with more side effects. However, in their study design, a larger patient population consisting of 80 symptomatic patients was included, 2 injections of PRP with 1-month interval were done, and a longer followup duration for up to 12 months using magnetic resonance arthrography and multiple pain and disability scoring systems was used [19].

There are many strong points concerning our study. First, the sample of the patients had the same diagnosis, and everybody received PRP injection. Shoulder functions and pain scores were collected at frequent time points after injection, allowing for an increased likelihood that subtle changes may be noticed. Therefore, the primary hypothesis is accepted. This study succeeded in demonstrating that those patients receiving PRP injections have decreased pain, improved healing properties, and superior functional outcomes.
The limitation of the study was the relatively short duration of follow-up with the need to involve a larger number of patients for a longer follow-up period in future studies to validate our results.

From our practice, although we did not have a recurrence of symptoms in the 24 patients who showed complete satisfaction with the results of a single session of PRP injection all over the 24 weeks of follow-up, we felt that studies should be directed towards more than one injection for the same patient at fixed scheduled dates and longer follow-up duration to assess the maximum efficacy of the PRP injection specially in those patients who were only slightly satisfied with one injection results.

\section{Illustrative case}

\section{Clinical data:}

A 52-year-old female patient, right-sided dominant, presented by right shoulder pain of 14-month duration with the inability to fully abduct her right arm.

Dominant or non-dominant shoulder: Dominant.

Medical history: HCV positive.

Surgical history: Irrelevant.

Trauma history: Negative.

By clinical examination: Limitation in almost all shoulder movements mainly above head abduction.

Baseline (SPADI) score was calculated:

1. For the pain score, $32 / 50$ or $64 \%$

2. For the disability score, $62 / 80$ or $77.5 \%$

3. For the total score, $94 / 130$ or $72.3 \%$

\section{Right shoulder ultrasound examination was done:}

The supraspinatus tendon is diffusely thickened with diffuse hypo-echogenicity and indistinct fibrillar pattern suggesting supraspinatus tendinopathy (Fig. 7a): 


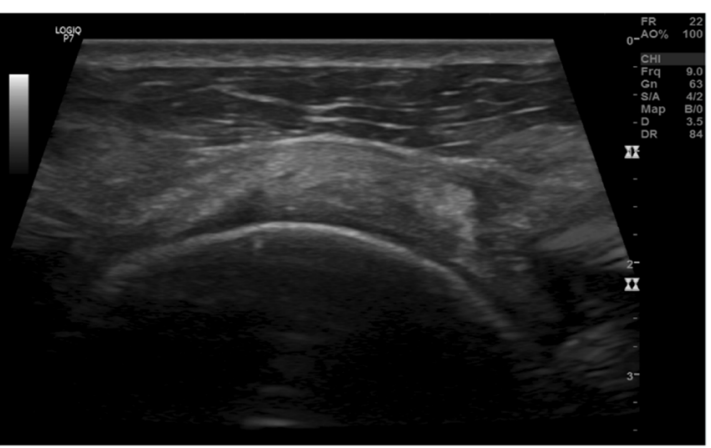

(A)

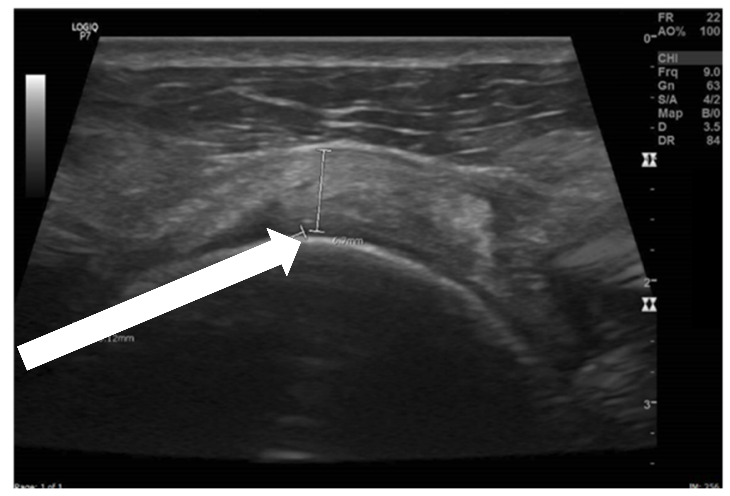

(B)

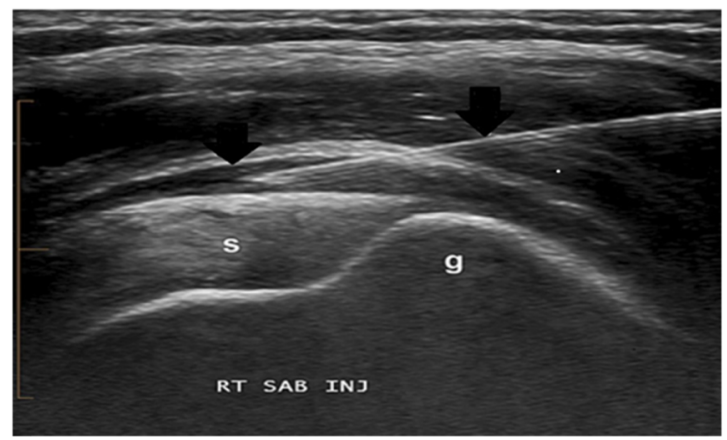

(C)

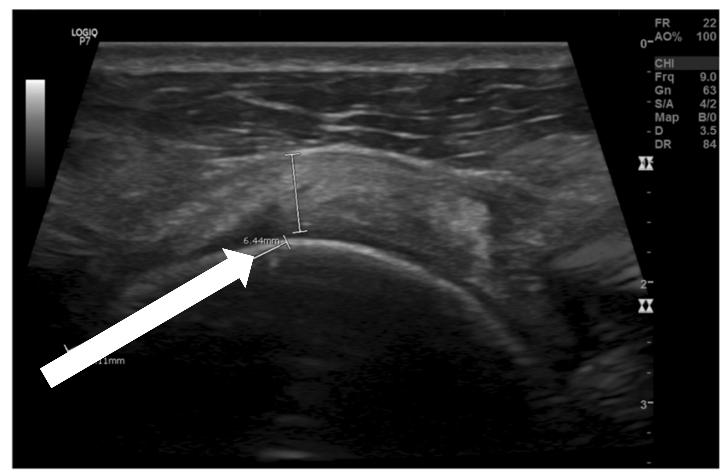

(D)
Fig. 7 a Right shoulder ultrasound with diffuse supraspinatus thickening and hypo-echogenicity. b Baseline supraspinatus tendon thickness (white arrow). c Ultrasound-guided injection of the right subacromion bursa (black arrows). d Follow-up supraspinatus tendon thickness after 24 weeks (white arrow)

1. Her baseline tendon thickness, $6.7 \mathrm{~mm}$ (Fig. 7b)

2. Ultrasound-guided PRP injection procedure was done at another appointment (Fig. 7c)

3. Assessment of her (SPADI) score after 24 weeks from the procedure date:

(a) For the pain score, $28 / 50$ or $56 \%$

(b) For the disability score, $48 / 80$ or $60 \%$

(c) For the total score, $76 / 130$ or $58.4 \%$

4. Assessment of her tendon thickness after 24 weeks from the procedure date, $6.4 \mathrm{~mm}$ (Fig. $7 \mathrm{~d}$ ).

\section{Conclusions}

The ultrasound-guided PRP injection for supraspinatus tendinopathy cases is a safe, cheap, and easily prepared outpatient procedure which showed competitive, promising, and well-proved results when compared to other modality outcomes such as conventional surgeries, arthroscopic procedures, and physiotherapy. PRP has the potential to heal the muscle-tendon unit of the rotator cuff at the level of degenerative tissues and may be a primary nonsurgical treatment modality for RCT.

We recommend that the future studies should have more concentration on the mechanism of action of PRP on tendinopathy that remains a challenge and deserves ongoing investigation. Furthermore, a long-term followup is recommended using ultrasound to assess the changes occurring in the injected tendon following the injection of PRP.

It deserves our attention to its value and efficacy for the sake of the patient as a minimally invasive procedure providing better quality of life.

\section{Abbreviations}

PRP: Platelet-rich plasma; US: Ultrasound; SPADI : Shoulder Pain and Disability Index; GFs: Growth factors; VEGF: Vascular endothelial growth factor

\section{Acknowledgements}

Not applicable.

\section{Consent to participate}

The whole procedures are explained in details to the patients, and they received detailed written information which explains the entire procedure and any possible risks that may happen (a written informed consent was obtained). 


\section{Authors' contributions}

GN performed the ultrasound examination for all patients included in the study and assisted in clinical evaluation of cases. Also, he assisted in the preparation of the platelet-rich plasma for each patient and performed ultrasound-guided injection of PRP for patients included in the study. MS performed follow-up ultrasound for all patients at the selected follow-up periods; also, he assisted in data collection and result analysis and played a major role in writing the manuscript. DM performed full clinical evaluation and examination for all patients included in the study and assisted in preparation of the platelet-rich plasma for each patient and performed full clinical assessment for all patients in the post-operative follow-up periods. The authors read and approved the final manuscript.

\section{Funding}

No funding was obtained for this study.

\section{Availability of data and materials}

All data and material of this article are readily available.

\section{Ethics approval and consent to participate}

This study was approved by the Research Ethics Committee of the Faculty of Medicine Ain Shams University together with departmental approval from the ethics committee of the Radiology Department of Ain Shams University. The ethics committee reference number is not applicable in this study as the protocol of this study was prepared by 2016 and we started patient enrollment and data collection by 2017-2018 which at such time the ethics committee reference number system was not established in our institute. Also, we did not make any changes in the protocol or methodology throughout the period of the study from the time of ethics committee approval and this technique is a well-established technique worldwide. All procedures performed in the study involving human participants were in accordance with the ethical standards of the institutional and/or national research committee and with the 1964 Helsinki Declaration and its later amendments or comparable ethical standards.

\section{Consent for publication}

All authors of this manuscript give a consent to the EJRNM to publish this article.

\section{Competing interests}

None.

\section{Author details}

${ }^{1}$ Radiology Department, Ain Shams University, Cairo, Egypt. ${ }^{2}$ Anesthesia, ICU and Pain Management Department, Ain Shams University, Cairo, Egypt.

Received: 4 May 2020 Accepted: 9 June 2020

Published online: 23 June 2020

\section{References}

1. Kuijpers T, van der Windt DA, van der Heijden GJ, Twisk JW, Ver-gouwe Y, Bouter LM. A prediction rule for shoulder pain related sick leave: a prospective cohort study. BMC Musculoskelet Disord 2006;7:97. https://doi. org/https://doi.org/10.1186/1471-2474-7-97

2. Ostor AJ, Richards CA, Prevost AT, Speed CA, Hazleman BL (2005): Diagnosis and relation to general health of shoulder disorders presenting to primary care. Rheumatology (Oxford, England); 44(6):800-805. https://doi.org/https:// doi.org/10.1093/rheumatology/keh598

3. Saladin KS (2016) Human anatomy, fifth edn. McGraw Hill, New York City, NY

4. Via AG, De Cupis M, Spoliti M and Oliva F (2013): Clinical and biological aspects of rotator cuff tears. Muscles, Ligaments and Tendons Journal, 3(2), https://doi.org/https://doi.org/10.11138/mltj/2013.3.2.070

5. Dill T (2008): Contraindications to magnetic resonance imaging: noninvasive imaging. Heart; 94(7):943-948. https://doi.org/https://doi.org/10. 1136/hrt.2007.125039

6. Longo UG, Franceschi F, Berton A, Maffulli N, Droena V (2012): Conservative treatment and rotator cuff tear progression. Medicine and sport science; 57: 90-99. https://doi.org/https://doi.org/10.1159/000328910

7. Chahal J, Van Thiel GS, Mall N, Heard W, Bach BR, Cole BJ, Nicholson GP, Verma NN, Whelan DB, Romeo AA (2012): The role of platelet-rich plasma in arthroscopic rotator cuff repair: a systematic review with quantitative synthesis. Arthroscopy: the journal of arthroscopic \& related surgery: official publication of the Arthroscopy Association of North America and the International Arthroscopy Association; 28(11):1718-1727. https://doi.org/ https://doi.org/10.1016/j.arthro.2012.03.007

8. Sanchez M, Anitua E, Orive G, Mujika I, Andia I (2009): Platelet-rich therapies in the treatment of orthopaedic sports injuries. Sports medicine (Auckland, NZ); 39(5):345-354. https://doi.org/https://doi.org/10.2165/00007256200939050-00002

9. De Vos RJ, Weir A, van Schie HT, et al (2010). Platelet-rich plasma injection for chronic Achilles tendinopathy: a randomized controlled trial. JAMA. 2010;303:144-149. https://doi.org/10.1001/jama.2009.1986

10. Sengodan VC, Kurian S, Ramasamy R (2017): Treatment of partial rotator cuff tear with ultrasound-guided platelet-rich plasma. Journal of clinical imaging science; 7:32. https://doi.org/https://doi.org/10.4103/jcis.jcis_26_17

11. Kirkley A, Griffin S, Dainty K (2003): Scoring systems for the functional assessment of the shoulder. Arthroscopy: the journal of arthroscopic \& related surgery: official publication of the Arthroscopy Association of North America and the International Arthroscopy Association; 19(10):1109-1120. https://doi.org/https://doi.org/10.1016/..arthro.2003.10.030

12. Hall MP, Band PA, Meislin RJ, Jazrawi LM, Cardone DA (2009): Platelet-rich plasma: current concepts and application in sports medicine. The Journal of the American Academy of Orthopaedic Surgeons; 17(10):602-608. https:// doi.org/https://doi.org/10.5435/00124635-200910000-00002

13. Noud PH, Esch J (2013): Complications of arthroscopic shoulder surgery. Sports Med Arthrosc Rev;21(2):89-96. https://doi.org/https://doi.org/10.1097/ jsa.0b013e31829006fo

14. Ilhanlil, Guder N, Gul M (2015): Platelet-rich plasma treatment with physical therapy in chronic partial supraspinatus tears. Iran Red Crescent Med J; 17(9):e23732. https://doi.org/https://doi.org/10.5812/ircmj.23732

15. Mautner K, Colberg RE, Malanga G, Borg-Stein JP, Harmo KG, Dharamsi AS, Chu S, Homer P (2013): Outcomes after ultrasound-guided platelet-rich plasma injections for chronic tendinopathy: a multicenter, retrospective review. PM\&R: the journal of injury, function, and rehabilitation; 5(3):169-175. https://doi.org/https://doi.org/10.1016/.jpmrj.2012.12.010

16. Randelli P, Arrigoni P, Ragone V, Aliprandi A, Cabitza P (2011): Platelet rich plasma in arthroscopic rotator cuff repair: a prospective RCT study, 2-year follow-up. J Shoulder Elb Surg; 20 (4):518-528. https://doi.org/https://doi. org/10.1016/j.jse.2011.02.008

17. Saltzman BM, JainA, CampbellKA, Mascarenhas R, Romeo AA, Verma NN, Cole BJ (2016): Does the use of platelet-rich plasma at the time of surgery improve clinical outcomes in arthroscopic rotator cuff repair when compared with control cohorts? A systematic review of meta-analyses. Arthroscopy;32:906-918 https://doi.org/https:/doi.org/10.1016/j.arthro.2015.10.007

18. Parada SA, Dilisio MF, Kennedy CD (2015): Management of complications after rotator cuff surgery. Current reviews in musculoskeletal medicine; 8(1): 40-52. https://doi.org/https://doi.org/10.1007/s12178-014-9247-6

19. Schwitzguebel AJ, Koko FC, Tirefort J et al (2019) (2019): efficacy of plateletrich plasma for the treatment of interstitial supraspinatus tears: a doubleblinded, randomized controlled trial. Am J Sports Med 47(8):1885-1892. https://doi.org/10.1177/0363546519851097 Epub 2019 Jun 4

\section{Publisher's Note}

Springer Nature remains neutral with regard to jurisdictional claims in published maps and institutional affiliations. 